\title{
Percepção dos Usuários do Sistema Único de Saúde frente a atuação do nutricionista
}

\author{
Nutritionist Activity in Primary Health Care: Perception of \\ Users of the Single Health System
}

Emanuella Magagna Amaro Pinto'; Daniele Faria Moreira'; Cinthia Aparecida Costa Rabêlo'; Érika Aparecida Pereira Azevedo;: Brunna Sullara Vilela'; Marco Antônio Olavo Pereira'

${ }^{1}$ Centro Universitário do Sul de Minas (UNIS-MG) - Varginha/MG, Brasil.

\section{Resumo}

Introdução: $O$ conhecimento da população em relação às práticas profissionais e atendimento oferecido na atenção básica possibilitam que a avaliação quanto aos serviços prestados seja benéfica para a melhoria do sistema. Conhecendo as atribuições dos nutricionistas, a população compreende o quão fundamental é a presença desse profissional na atenção básica à saúde. Objetivo: Analisar a percepção dos usuários do Sistema Único de Saúde frente a atuação do nutricionista da Atenção Primária à Saúde. Metodologia: Esta pesquisa foi realizada em vinte Unidades Básicas de Saúde, na cidade de Varginha- MG, composta por usuários da rede de atenção primária à saúde, entre 18 e 70 anos e em atendimento ambulatorial. O questionário aplicado apresenta conteúdo baseado nas ações rotineiras do nutricionista que atua na atenção básica. Resultados: $A$ análise da pesquisa demonstrou que os usuários acreditam que a alimentação é um fator de prevenção para diversas doenças e, com isso, a busca pelo nutricionista está aumentando, considerando assim o reconhecimento pelo profissional. Conclusão: Portanto, relacionando a atuação do profissional com a opinião dos usuários, o reconhecimento deste na área de alimentação e nutrição tem sido exercida de modo positivo, atendendo as necessidades da população e conseguindo demonstrar o quão importante é a sua presença dentro da atenção básica.

Palavras chave: Sistema Único de Saúde; Ciências da Nutrição; Atenção Primária à Saúde. 


\begin{abstract}
Introduction: The knowledge of the population about professional practices and care offered in basic care, make it possible for the evaluation of the services rendered to be beneficial for the improvement of the system. Knowing the attributions of nutritionists, the population understands how fundamental the presence of this professional in basic health care is. Objective: Analyze the perception of users of the Unified Health System regarding the performance of the Primary Health Care nutritionist. Methodology: This research will be carried out in twenty Basic Health Units in the city of Varginha-MG, primary health care, between 78 and 70 years and outpatients. The applied questionnaire presents content based on the routine actions of the primary care nutritionist. Results. The analysis of the research demonstrated that the users believe that the feeding is a factor of prevention for several diseases, and with that the search for the nutritionist is increasing, thus considering the recognition by the professional. Conclusion: Therefore, relating the performance of the professional with the opinion of the users, the knowledge of the same in the area of food and nutrition is being exercised in a positive way, meeting the needs of the population and demonstrating how important is their presence within the attention basic.
\end{abstract}

Keywords: Unified Health System; Nutritional Sciences; Primary Health Care.

Recebido em: 14-08-2018 Publicado em:29-10-2020

\section{Autor correspondente}

Daniele Faria Moreira

Endereço: R. Cel. José Alves, 256 Vila Pinto.

CEP 37010-540 - Varginha (MG), Brasil.

Email: daniele.moreira@professor.unis.edu.br

\section{Introdução}

A criação do Sistema Único de Saúde (SUS) tem como intuito modificar a assistência desigual à saúde dos indivíduos, estrategicamente criada para implementar ações na área da saúde, tornando obrigatório o atendimento gratuito para toda a população?.

A Atenção Primária, também conhecida por Atenção Básica, deve garantir o acesso universal aos serviços de saúde, considerada a porta de entrada dos usuários ao sistema, deve solucionar grande parte dos problemas e necessidades da população, por meio da promoção, proteção, diagnóstico, tratamento e manutenção da saúde ${ }^{2,3}$.

Como estratégia orientadora da Atenção Básica, foi criado o Programa Saúde da Família (PSF), conhecido hoje por Estratégia Saúde da Família (ESF), a qual tem a intenção de reorganizar a rede de assistência básica, ou seja, substituir o modelo tradicional por outro com novas práticas e equipe multidisciplinar, assegurando à população uma oferta de serviços contínuos pelos profissionais que compõe as equipes de ESF, fortalecendo, assim, os princípios de acessibilidade, integralidade, equidade e universalidade do SUS 4 . 
Para melhorar a qualidade da Atenção Básica à Saúde e fortificar a Estratégia Saúde da Família, o Ministério da Saúde criou os Núcleos Ampliados de Saúde da Família e Atenção Básica (NASF- AB). O NASF- AB tem o objetivo de aumentar a abrangência das ações de atenção básica à saúde como, por exemplo, os profissionais que devem atuar em parceria com as equipes de Saúde da Família. Um dos profissionais inseridos nesse programa é o nutricionista ${ }^{5}$.

Conhecer o perfil e o processo de formação dos profissionais que atuam na atenção básica fortalece o trabalho multiprofissional, e avança para 0 desenvolvimento de ações interdisciplinares que beneficia a família como o centro de atenção. Assim, é de relevância apontar a importância de o nutricionista integrar a atenção básica à saúde, considerando que sua ausência pode resultar em execuções parciais das ações relacionadas à alimentação e nutrição, área considerada fundamental para a promoção da saúde $e^{6-8}$.

Diante da influência da alimentação sobre o processo saúde-doença, os NASFAB propõem algumas ações como capacitar a equipe de saúde da família e participar de ações vinculadas aos programas de controle e prevenção dos distúrbios nutricionais, estimular o consumo dos alimentos saudáveis produzidos regionalmente, elaborar em conjunto com as equipes de saúde da família rotinas de atenção nutricional e atendimento para doenças relacionadas à alimentação e nutrição. Essas atividades ampliam a qualidade dos planos de intervenção, em especial, quanto às doenças crônicas não transmissíveis, no crescimento e desenvolvimento na infância, e gestação, comprovando que a promoção de hábitos alimentares saudáveis constitui elemento essencial em todas as fases da vida. Assim, as ações de Alimentação e Nutrição integram o compromisso da Atenção Básica de garantir o essencial à população, a segurança alimentar e nutricional?

Além de princípios como integralidade e universalidade dentro do sistema único de saúde, existe ainda a diretriz que diz respeito à participação da comunidade. $O$ controle social é a demonstração mais viva da participação da sociedade nas decisões do Estado no interesse geral. Com o incentivo à ação popular junto ao fortalecimento vindo do SUS, presume-se que o usuário apresenta competência para avaliar e intervir no próprio sistema ${ }^{10}$.

A participação crítica e livre dos indivíduos pode contribuir para aprimorar a promoção à saúde. O conhecimento das atribuições do nutricionista na atenção básica com o profissional apto para atuar na prevenção de agravos, como obesidade, hipertensão arterial sistêmica, Diabetes Melittus e a promoção da saúde em todos os ciclos e fases da vida, possibilitam que a avaliação quanto aos serviços prestados seja satisfatória para a melhoria do sistema ${ }^{11,12}$.

Considerando a importância do trabalho do profissional nutricionista perante a saúde individual e da população, o objetivo da pesquisa foi analisar a percepção dos usuários do Sistema Único de Saúde frente à atuação do nutricionista da Atenção Primária à Saúde.

\section{Metodologia}

Trata-se de um estudo transversal, realizado em vinte Unidades Básicas de Saúde localizadas na cidade de VarginhaMG, as quais contavam com a presença de nutricionistas. A amostra foi 
determinada por critério de conveniência e constituída de 65 usuários da rede de atenção primária à saúde, incluídos homens e mulheres com idade entre 18 e 70 anos, em atendimento ambulatorial de nutrição, no dia da coleta de dados. Foram assinados os Termos de Consentimento Livre e Esclarecido e, como critérios de exclusão, foram considerados os pacientes com idade menor que 18 anos e maior que 70 anos que não tinham realizado atendimento nutricional e não possuíam autonomia para responder o questionário.

Para a coleta de dados, foi aplicado um questionário (ANEXO 1) desenvolvido a partir do conhecimento sobre as ações rotineiras do nutricionista da atenção primária de acordo com os cadernos de atenção básica do Ministério da Saúde ${ }^{13}$ com o apoio de um nutricionista atuante na rede municipal de Varginha, MG.

A coleta foi realizada no período de março e abril de 2018, etapa na qual o participante foi submetido a uma entrevista de maneira individual. 0 questionário foi composto por questões sociodemográficas como sexo, estado civil, etnia, grau de instrução e, também, a percepção da atuação do nutricionista, motivo e tempo do acompanhamento nutricional, ações realizadas pelo profissional e satisfação pelo trabalho que é feito com os usuários.

Os dados foram tabulados no software Excel e, posteriormente, foi aplicada a técnica de estatística descritiva (frequência, absoluta e relativa, média e desvio padrão).

Os questionários foram aplicados de forma anônima, facultados de maneira aleatória para preservar o sigilo da identificação dos usuários entrevistados. A pesquisa foi submetida e aprovada pelo Comitê de Ética em pesquisa do Centro Universitário do Sul de Minas, sob CAAE de número 83429218.0.0000.5111.

Em nenhum momento do estudo houve conflito de interesses entre os autores e suas instituições.

\section{Resultados}

A média de idade dos participantes foi de $46,4 \pm 74,62$ anos, e a maioria eram mulheres $87,7 \%$ ( $n=57$ ). Sobre as características sociodemográficas dos entrevistados, foi observado que a maioria é casada (66,2\%), considera-se parda $(55,4 \%)$ e $30,8 \%$ possui ensino médio completo.

Na tabela 1, é apresentado o tempo de acompanhamento nutricional dos participantes. A maior parte dos pacientes $46 \%(n=30)$ declara estar em acompanhamento recente, menos de um mês. Por outro lado, apenas 14\% ( $n=9)$ estão em tratamento há mais tempo, um ano ou mais. 
TABELA 1- Tempo de acompanhamento nutricional dos pacientes das Unidades de Saúde de Varginha

\begin{tabular}{ccc}
\hline Acompanhamento nutricional dos participantes & $\mathrm{N}$ & $\%$ \\
\hline Menos de 1 mês & 30 & 46 \\
2 a 4 meses & 10 & 15 \\
6 meses ou mais & 16 & 25 \\
Mais de um ano & 09 & 14 \\
\hline
\end{tabular}

Os motivos declarados pelos participantes que os fizeram buscar o acompanhamento com o profissional nutricionista estão apresentados na Tabela 2. Nota-se que a maior parte dos usuários, 36,9\% ( $n=24)$, procurou 0 atendimento devido ao grau de sobrepeso e 16,9\% (n=11) e 15,4\% ( $n=10)$ buscaram por motivos de Diabetes Mellitus e hipertensão arterial sistêmica, respectivamente.

TABELA 2- Motivos para buscar o acompanhamento nutricional nas Unidades de Saúde de Varginha

\begin{tabular}{lll}
\hline Motivos & $\mathrm{N}$ & $\%$ \\
\hline Auriculaterapia & 01 & 1,5 \\
Diabetes mellitus & 11 & 16,9 \\
Esofagite & 01 & 1,5 \\
Ganho de massa corporal & 01 & 1,5 \\
Ganho de peso & 04 & 6,2 \\
Gestação & 04 & 6,2 \\
Hipercolesterolemia & 03 & 4,6 \\
Hipertensão & 10 & 15,4 \\
Hipertriglicemia & 01 & 1,5 \\
Lúpus & 01 & 1,5 \\
Menopausa & 01 & 1,5 \\
Reeducação alimentar & 03 & 4,6 \\
Sobrepeso & 24 & 36,9 \\
\hline
\end{tabular}


Na Tabela 3, está representado o conhecimento dos usuários do SUS quanto às ações que os nutricionistas realizam nas Unidades de Saúde. Destaca-se que $89,2 \% \quad(n=58)$ reconhecem ser papel do nutricionista prestar atendimento para pessoas com doenças crônicas não transmissíveis (DCNT) mas, por outro lado, 78,5\% ( $n=51)$ dos entrevistados não sabia que 0 nutricionista deveria atender crianças de O a 6 meses. Já em relação às ações que os entrevistados acreditam que deveriam ser realizadas, $66,2 \%$ dos usuários afirmaram que o atendimento infantil deveria ser realizado nas unidades de saúde, enquanto 56,9\% não julgaram necessária a realização de oficinas pelos profissionais.

TABELA 3 - Conhecimento dos usuários do SUS sobre as atividades realizadas pelos nutricionistas nas Unidades de Saúde

\begin{tabular}{|c|c|c|}
\hline & $\begin{array}{l}\text { Ações que os } \\
\text { usuários } \\
\text { acreditam ser } \\
\text { realizadas pelo } \\
\text { nutricionista } \\
\text { (\%) }\end{array}$ & $\begin{array}{l}\text { Ações que os } \\
\text { usuários } \\
\text { acreditam que } \\
\text { deveriam ser } \\
\text { realizadas pelo } \\
\text { nutricionista (\%) }\end{array}$ \\
\hline & SIM & SIM \\
\hline Atendimento domiciliar & 16,9 & 52,3 \\
\hline Atendimento infantil & 33,8 & 66,2 \\
\hline Atendimento para adolescentes & 38,5 & 63,1 \\
\hline Atendimento para adultos de 20 a 60 anos & 81,5 & 47,7 \\
\hline Atendimento para bebês de 0 a 6 meses & 21,5 & 55,4 \\
\hline Atendimento para DCNT & 89,2 & 52,3 \\
\hline Atendimento para desnutridos & 55,4 & 44,6 \\
\hline Atendimento para gestantes & 44,6 & 63,1 \\
\hline Atendimento para idosos acima de 60 anos & 72,3 & 52,3 \\
\hline Atendimento para obesos & 70,8 & 53,8 \\
\hline Atendimentos em grupo & 36,9 & 49,2 \\
\hline Educação em saúde & 58,5 & 49,2 \\
\hline Oficinas & 21,5 & 43,1 \\
\hline Orientação para pós-desmame & 32,3 & 44,6 \\
\hline Orientações sobre Aleitamento materno & 24,6 & 60 \\
\hline Palestras & 40 & 56,9 \\
\hline Prescrição de Planos Alimentares & 72,3 & 46,2 \\
\hline
\end{tabular}

- A tabela 4 apresenta a opinião dos usuários do SUS sobre a possibilidade de as orientações nutricionais poderem ser dadas por outros profissionais. Os dados mostraram que 73\% ( $n=47)$ dos usuários acreditam que apenas o nutricionista é capacitado para oferecer orientações nutricionais, porém 9\% $(n=6)$ acreditam que endocrinologistas e profissionais de educação física também podem orientar os usuários. 
TABELA 4 - Opinião dos usuários sobre os profissionais que poderiam realizar as orientações nutricionais.

\begin{tabular}{llc}
\hline Motivos & $\mathrm{N}$ & $\%$ \\
\hline Endocrinologista & 06 & 9,0 \\
Enfermeiro & 02 & 3,0 \\
Médico & 04 & 6,0 \\
Nutricionista & 47 & 73,0 \\
Educador físico & 06 & 9,0 \\
\hline
\end{tabular}

A tabela 5 apresenta o grau de satisfação dos usuários em relação ao atendimento com o nutricionista na atenção básica, e que 98,5\% ( $n=64)$ relataram satisfação com o atendimento recebido.

TABELA 5 - Grau de satisfação dos entrevistados que receberam atendimento nutricional na atenção básica de Varginha.

\begin{tabular}{lll}
\hline Grau de satisfação & $\mathrm{N}$ & $\%$ \\
\hline Muito satisfeito & 46 & 70,8 \\
Satisfeito & 18 & 27,7 \\
Indiferente & 00 & 0,0 \\
Insatisfeito & 01 & 1,5 \\
Muito insatisfeito & 00 & 0,0 \\
\hline
\end{tabular}

\section{Discussão}

Este estudo mostrou que a maioria dos participantes reconhece ser papel do nutricionista prestar atendimento para pessoas com doenças crônicas não transmissiveis (DCNT) e acreditam que apenas o nutricionista é capacitado para oferecer orientações nutricionais. Também relataram estar satisfeitos com - atendimento do nutricionista na atenção básica. Esses dados podem ser resultados das ações de alimentação e

Revista Conexão Ciência I Vol. 16 I N² 2 I 2021 nutrição no Brasil iniciadas antes do SUS e que se consolidaram a partir do SUS, e já completaram 30 anos de existência ${ }^{14}$.

Além disso, com o aumento de doenças como diabetes, obesidade, dislipidemias, hipertensão e câncer, a população despertou para um maior interesse sobre a importância da alimentação adequada. O nutricionista desempenha um papel de grande importância na promoção e prevenção da saúde e o tratamento de doenças relacionadas à alimentação faz 
com que a busca pelo seu trabalho seja cada vez maior ${ }^{15}$.

Como observado no presente estudo, o tempo de acompanhamento nutricional, recentemente tem sido amplificado, demonstrando que a nutrição tem sido solicitada para melhoria na qualidade de vida e saúde. E, ainda, que o sobrepeso, diabetes e hipertensão foram determinantes para a procura deste profissional. De acordo com a Organização Mundial da Saúde, a obesidade é considerada uma epidemia mundial relacionada pela atividade física e perfil alimentar ${ }^{16}$. No Brasil, mais da metade da população brasileira está com excesso de peso ${ }^{17}$.

A pesquisa mostrou que 36,9\% dos entrevistados que buscaram atendimento nutricional o fizeram por causa do sobrepeso. É importante ressaltar que o excesso de peso afeta o metabolismo dos lipídios e glicose, pressão arterial, aumenta as citocinas inflamatórias e está associado com doenças cardiovasculares ${ }^{18-20}$. Além disso, ocorrem prejuízos econômicos e sociais associados à mortalidade e à morbidade precoce da obesidade. Dificuldades no âmbito social e ocupacional, menor produtividade, riscos à saúde, baixa autoestima são alguns dos prejuízos que a obesidade provoca os quais afetam a qualidade de vida dos indivíduos ${ }^{21}$.

Neste estudo, 16,9\% dos usuários eram diabéticos e 15,4\% hipertensos. Conforme a pesquisa realizada, no ano de 2017, pela Vigilância de Fatores de Risco e Proteção para Doenças Crônicas por Inquérito Telefônico (VIGITEL), 8,9\% dos brasileiros são portadores de Diabetes Melittus e 25,7\% são hipertensos. Para prevenção das doenças crônicas não transmissiveis, a alimentação é considerada o centro da promoção da saúde por meio da relação, hábitos alimentares saudáveis e a prática de atividade física ${ }^{22-24}$.

O nutricionista da atenção primária possui um papel vital à promoção e proteção da saúde ${ }^{25}$. Uma das ações dentro das unidades de saúde são os atendimentos a pessoas com doenças crônicas não transmissíveis e, neste estudo, foi considerada uma atividade relevante para os usuários. Uma das estratégias para a prevenção dessas doenças é a alimentação adequada ${ }^{17}$.

Além das DCNT's, é um dos deveres do nutricionista prestar atendimento infantil, pois essa é uma parcela da população que está em crescimento quando o assunto tratado é obesidade e desnutrição ${ }^{26}$. Dos entrevistados, 66,2\% acreditam que o nutricionista deveria prestar atendimento infantil, todavia isso é uma ação que já ocorre, indicando que falta conhecimento sobre as atribuições do profissional. Uma revisão bibliográfica realizada por Cervato, Vincha e Santiago (2016) constatou que a abordagem nutricional com as crianças prevalece nos serviços de saúde, e o nutricionista é o profissional responsável pela condução de ações educativas ${ }^{27}$. Além da educação nutricional para as crianças, as intervenções precisam ser feitas junto aos pais. A OMS relata que a urbanização, disponibilidade de "fastfood", aumento do tempo diante da televisão e videogames e menor tempo para atividade física são fatores que predispõe crianças à obesidade ${ }^{28}$. 0 comportamento alimentar e físico da família é modelo para seus filhos, a determinação de alimentos disponíveis em quantidade e qualidade e um agradável ambiente emocional são condutas que amenizam a obesidade ${ }^{29}$.

A preocupação com a alimentação tornou-se um hábito constante em diferentes parcelas da população. É um dos papéis do nutricionista auxiliar as 
pessoas a modificarem seus hábitos alimentares, por meio da assistência a indivíduos e grupos populacionais. Aumentar os conhecimentos nutricionais é uma estratégia eficaz para a promoção de práticas alimentares saudáveis, que asseguram escolhas alimentares conscientes. 0 estudo dos conhecimentos nutricionais permite analisar melhor o que a população sabe sobre a alimentação para, enfim, poder melhorar a atuação em programas de educação nutricional ${ }^{29}$.

Este estudo revelou, ainda, que os entrevistados acreditam ser o nutricionista o profissional adequado para orientar sobre alimentação. Segundo Casas-Agustench, MegiasRangil \& Babio (2020). incorporar a presença de nutricionistas na atenção primária melhora a qualidade de vida, reduz a demanda por atendimento médico especializado, capacita as pessoas ao autocontrole de suas doenças crônicas e reduz a necessidade de internação ${ }^{30}$.

As mudanças culturais, socioeconômicas e tecnológicas resultam em transformações das características da alimentação. Hábitos danosos à saúde levam a ocorrência de problemas como hipertensão, diabetes, obesidades e doenças cardiovasculares, advindos do consumo excessivo de produtos industrializados e pelo uso de aditivos químicos. O nutricionista tem um papel fundamental para mudar essa realidade. É por meio da promoção de hábitos saudáveis que se proporciona uma boa saúde para a população. O profissional atua de modo consciente, coletivo, promovendo a aproximação das pessoas com a alimentação, lutando pelo direito destas por uma alimentação adequada ${ }^{31}$. As ações do profissional, junto à determinação dos indivíduos, satisfazem ambas as relações, e os entrevistados encontram-se satisfeitos com atendimento nutricional. De maneira semelhante, pode ser visto no estudo realizado por Pacheco (2009), no município de Porto Alegre, onde os usuários também se encontram satisfeitos, citando que os profissionais do município são pessoas responsáveis por um excelente trabalho e que possuem grande preocupação com a população tratada 32 .

Deve-se ressaltar que este estudo apresenta limitações quanto à sua amostra, que ao se apresentar em número reduzido, pode não representar toda a comunidade de usuários dos serviços de atenção básica de Varginha MG. Entretanto, ele fornece ferramentas importantes para pensar a respeito desse ponto de vista do usuário. Além disso, o tema é escasso na literatura, são necessários mais estudos na área.

É preciso destacar, também, o perfil dos participantes da pesquisa. Nesta evidenciou-se um predomínio do sexo feminino, e, decorrente de costumes, as mulheres preocupam-se mais com os hábitos saudáveis ${ }^{33}$. Oliveira, Lorenzatto e Souza (2010) falam que a maioria das entrevistadas pertencia ao gênero feminino e, ainda, consideram os homens resistentes na procura por serviços de saúde 34

Analisando o grau de escolaridade da população, observou-se que a maior parte possui ensino médio completo, segundo Celestino \& Neves (2009), o alto nível de escolaridade contribui para maior acessibilidade de informações ${ }^{35}$. De acordo com Riveira e Souza (2007), a baixa escolaridade pode influenciar de forma negativa a alimentação devido à falta de conhecimento sobre os nutrientes e suas funções ${ }^{36}$. A escolaridade ensino fundamental $\left(7^{\circ}\right.$ ao $5^{\circ}$ ano) incompleto 
apresentou relevância no resultado, ou seja, verificou-se que a procura por informações para uma boa qualidade de vida, atualmente, tem superado os níveis de escolaridade.

Diante de todo o exposto, é importante a integração dos nutricionistas em todas as ações desenvolvidas pela atenção básica aos cuidados à saúde em diferentes fases ${ }^{37}$. O estudo demonstrou que os usuários acreditam que a alimentação é um fator de prevenção para diversas doenças, e que a busca pelo nutricionista tem aumentado.

\section{Conclusão}

Conclui-se que ao relacionar a atuação do profissional nutricionista com a opinião dos usuários, verifica-se que o reconhecimento deste na área de alimentação e nutrição tem sido exercido de modo positivo, uma vez que este atende as necessidades da população e consegue demonstrar o quão importante é a sua presença dentro da atenção básica.

Os resultados apresentados constituem um conjunto de ações que apoiam o nutricionista na sua meta de promoção da saúde aos usuários do sistema.

\section{Declaração de conflito de interesses}

○ autor do artigo afirma que não se encontram em situações de conflito de interesse que possam influenciar o desenvolvimento do trabalho, tais como emissão de pareceres, propostas de financiamento, promoções ou participação em comitês consultivos ou diretivos, participação em estudos clínicos e/ou experimentais subvencionados; atuação como palestrante em eventos patrocinados; participação em conselho consultivo ou diretivo; comitês normativos de estudos científicos; recebimento de apoio institucional; propriedade de ações; participação em periódicos patrocinados, assim como qualquer relação financeira ou de outra natureza com pessoas ou organizações que possam influenciar o trabalho de forma inapropriada.

\section{Referências}

1 - BRASIL. Ministério da Saúde. SUS de A a Z. Disponível em:

<http://portal.saude.gov.br/portal/saude/ cidadao/default.cfm>. Acesso em: 16 de julho de 2018.

2 - SÃO PAULO. Política de apoio à atenção básica no SUS/SP. São Paulo: Secretaria Municipal da Saúde.

Disponível em: <http://www.saude.sp.gov.br/resources/ gestor/projetos/politica_de_apoio_a_ate ncao_basica_no_estado_de_sp.pdf.>. Acesso em 16 de julho de 2018.

3 - BRASIL. Ministério da saúde. Secretaria de Atenção À Saúde. Departamento de Atenção Básica e Saúde da Família. Política Nacional de Atenção Básica. Brasília, DF: MS, 2006. Disponível em: <http://189.28.128.100/dab/docs/publicac oes/geral/pnab.pdf>. Acesso em: 16 out. 2017.

\section{4 - FUNDAÇÃO OSWALDO CRUZ. Saúde da família. [S.d.] Disponível em: $<$ https://pensesus.fiocruz.br/saude-da- familia>. Acesso em: 10 set. 2017.}


5 - BRASIL. Ministério da Saúde. Portaria n 154 de 24 de janeiro de 2008. Trata dos Núcleos de Apoio à Saúde da Família NASF. Disponível em: <http://189.28.128.100/dab/docs/legislaca o/portaria154_24_01_08.pdf>. Acesso em: 16 de julho de 2018.

6 - ASSIS, A. M. O.; SANTOS, S. M. C.; FREITAS, M. C. S.; SANTOS, J. M.; SILVA, M. C. M. O Programa Saúde da Família: contribuições para uma reflexão sobre a inserção do nutricionista na equipe multidisciplinar. Revista Nutri, v.15, n.3, p.255-266, 2002.

7 - PÁDUA, J. G.; BOOG, M. C. F. Avaliação da inserção do nutricionista na Rede Básica de Saúde dos municípios da Região Metropolitana de Campinas. Revista Nutr v.19, n.4, p. 412424, 2006.

8 - SANTOS, A. C. A inserção do nutricionista na Estratégia de Saúde da Família: o olhar de diferentes trabalhadores da saúde. Família Saúde e Desenvolvimento, v.7, n.3m p.257-265, 2005.

9 - MATTOS, P. F.; DOS SANTOS, A. N. A importância da atuação do nutricionista na Atenção Básica à Saúde. Revista Práxis, v. 1, n. 2, 2017.

10 - CREVELIM, M. A. Participação da comunidade na equipe de saúde da família: é possível estabelecer um projeto comum entre trabalhadores e usuários? Ciência. saúde coletiva. Rio de Janeiro, v. 10, n. 2, p. 323-331, 2005.

11 - COTTA, R. M. M. et al. Reflexões sobre o conhecimento dos usuários no contexto do Programa de Saúde da Família: a lacuna entre o saber técnico e o popular. Physis: Revista de Saúde Coletiva. Rio de Janeiro, v. 18, n. 4, p. 745766, 2008.

12 - cosTA, K. A. O.; SANTANA, P. R. A importância e o papel do nutricionista na atenção básica em Vitória De Santo Antão / PE. Brasília, Tempus Actas de Saúde Coletiva,v. 5, n. 4, p. 67-85, 2011. Disponivel em: <http://www.tempusactas.unb.br/index. php/tempus/article/view/1058>. Acesso em: 16 out. 2017.

13 - CADERNOS DA ATENÇÃO BÁSICA. Obesidade. Cadernos da Atenção Básica n¹2. Ministério da Saúde. Brasília, 2006. Disponivel em: <http://dtr2004.saude.gov.br/dab/docu mentos/cardernos_ab/documentos/abc ad12.pdf> Acesso em: 23 jun. 2018.

14 - BORTOLINI, G. A.; OLIVEIRA, T.F.V.; SILVA, S. A.; SANTIN, R. C.; MEDEIROS, O.L. et al. Ações de alimentação e nutrição na atenção primária à Saúde no Brasil. Rev. Panamericana de Salud Pública, v. 44, p. e39, 2020.

15 - CONSELHO REGIONAL DE NUTRICIONISTA N 5 . Entidades de nutrição reforçam a valorização dos nutricionistas. 02 de Fevereiro de 2016. Disponível em: <http://crn5.org.br/entidades-de- 
nutricao-reforcam-valorizacao-dosnutricionistas/> Acesso em: 23 de Junho de 2018.

16 - WORLD HEALTH ORGANIZATION. Obesity: preventing and managing the global epidemic. World Health Organization, 2000.

17 - CAIVANO, S.; LOPES, R. F.; SAWAYA, A. L.; DOMENE, S.M.A; MARTINS, P.A. Conflitos de interesses nas estratégias da indústria alimentícia para aumento do consumo de alimentos ultraprocessados e os efeitos sobre a saúde da população brasileira. DEMETRA, v. 12, n. 2, p. 349-360, 2017.

18 - FARKHONDEH, T.; LLORENS, S.; POURBAGHER-SHAHRI, A.M.; ASHRAFIZADEH, M.; TALEBI, M.; SHAKIBAEI, M. et al. An Overview of the Role of Adipokines in Cardiometabolic Diseases. Molecules, v. 25, n. 5218, 2020.

19 - KOJTA, I.; CHACIŃSKA, M.; BŁACHNIOZABIELSKA, A. Obesity, Bioactive Lipids, and Adipose Tissue Inflammation in Insulin Resistance. Nutrients, v. 12, n. 5, p. 1305, 2020.

20 - YANOVSKI, J. A.; YANOVSKI, S. Z. Recentes avanços na pesquisa básica em obesidade. JAMA Brasil, v.2, n.4, p. 2822-2824, 2000.

21 - HIILAMO, A.; LALLUKKA, T.; MÄNTY, M.; KOUVONEN A. Obesity and socioeconomic disadvantage in midlife female public sector employees: a

Revista Conexão Ciência I Vol. 16 | N² 2 I 2021 cohort study. BMC Public Health, v. 17, n. 842, 2017.

22 - AZEVEDO L. M. E.; DE SOUZA, H. N. F.; DE FÁTIMA, M. F. Doenças crônicas não transmissiveis, risco e promoção da saúde: construções sociais de participantes do Vigitel. Revista Ciência \& Saúde Coletiva, v. 20, n. 3, 2015.

23 - VIGITEL. Pesquisa revela que diabetes no Brasil cresceu 61,8\% em dez anos. Disponivel em: <http://agenciabrasil.ebc.com.br/geral/n oticia/2017-04/pesquisa-revela-quediabetes-no-brasil-cresceu-618-em-dezanos> Acesso em: 23 jun. 2018.

24 - SOUZA, J. A. Conhecimentos Nutricionais, Reprodução e Validação do questionário. Tese (Mestrado em Saúde Pública) - Faculdade de Medicina, Universidade do Porto, 2009.

25 - O'CONNOR, R. K. SLATER, L.; BALL, A. Jones The tension between efficiency and effectiveness: a study of dietetic practice in primary care. J Hum Nutr Diet, v. 32, n. 2, p. 259-266, 2019.

\section{6 - ORGANIZAÇÃO PAN- AMERICANA DA} SAÚDE. Obesidade entre crianças e adolescentes aumentou dez vezes em quatro décadas revela novo estudo do Imperial College London e da OMS. 10 de Outubro de 2017. Disponível em: <https://www.paho.org/bra/index.php?o ption=com_content\&view=article\&id $=55$ 27:obesidade-entre-criancas-eadolescentes-aumentou-dez-vezes-emquatro-decadas-revela-novo-estudo-doimperial-college-london-e-da- 
oms\&ltemid=820 > Acesso em: 23 jun. 2018.

27 - CERVATO, A. M. M.; VINCHA, K. R. R.; SANTIAGO, D.A. Food and Nutrition Education as intervention practice: reflection and possible strengthening. Physis: Revista de SaúdeColetiva, v. 26, n. 1, p. 225-249, 2016.

28 - NAÇÕES UNIDAS. OMS lança novas diretrizes de combate a obesidade infantil no mundo. 06 de Abril de 2017. Disponível em: $<$ https://nacoesunidas.org/oms-lancanovas-diretrizes-de-combate-aobesidade-infantil-no-mundo/> Acesso em: 23 jun. 2018.

29 - RHEE K. E.; LAGO C. W.; ARSCOTT T. M.; MEHTA S. D.; DAVIS R. K. Factors associated with parental readiness to make changes for overweight children. Pediatrics, v. 116, n. 1, p. 94-101, 2005.

30 - CASAS-AGUSTENCH, P.; MEGIASRANGIL, I.; BABIO, N. Economic benefit of dietetic-nutritional treatment in the multidisciplinary primary care team. Nutrición Hospitalaria. v. 37, n.4, p. 863$874,2020$.

31 - CONSELHO FEDERAL DE NUTRIÇÃO.

Resolução Cfn No 599, de 25 de Fevereiro de 2018. Disponível em: <http://www.cfn.org.br/wpcontent/uploads/resolucoes/Res_599_20 18.htm> Acesso em: 23 de Junho de 2018.
32 - PACHECO, P.M.; Entendimentos e Percepções da Atuação do Nutricionista em Atenção Primária: uma visão a partir do olhar do usuário do SUS. (Trabalho de conclusão de curso), Universidade Federal do Rio Grande do Sul, 2009.

33 - OLIVEIRA, A. F.; LORENZATTO, S.; SOUZA, E. C. F. Perfil de Pacientes que procuram atendimento nutricional. Revista Salus, v. 2, n. 1, p. 1321, 2010.

34 - PORTO, M. C. V.; BRITO, I.C.; CALFA A. D. F.; AMORAS, M.; VILLELA N. B.; ARAÚJO, L. M. B. Perfil do obeso classe III do ambulatório de obesidade de um hospital universitário de Salvador. Arq Bras Endocrinol Metab Bahia, v. 1, n. 46, p. 668-673, 2006.

35 - CELESTINO, M.; NEVES, C. S. Perfil Socioeconômico e Estado Nutricional dos pacientes atendidos na Clínica de Nutrição da Faculdade União Das Américas. Seminário Científico de Nutrição, v. 1, n. 1, 2009.

36 - RIVERA, F. S. R.; SOUZA, E. M. T. Consumo Alimentar de Escolares de uma Comunidade Rural. Comunidade Ciência da Saúde. Faculdade de Ciências da Saúde. Universidade de Brasília. Brasília p. 111 - 119, 2007.

37 - PACHECO, P. M.; RAMOS, M. Nutritionists in primary health care: the user's viewpoint. Demetra: food, nutrition \& health, v. 9, n. 2, p. 483-501, 2014. 